\title{
Nijmegen breakage syndrome in two half sibs with peripheral T-cell lymphoma and cortical T-cell acute lymphoid leukemia
}

\author{
SVETLANA O. SHARAPOVA ${ }^{1}$ ELENA I. GOLOVATAYA², ELENA V. SHEPELEVICH ${ }^{2}$, \\ YULIYA E. MAREIKA ${ }^{3}$, IRINA E. GURYANOVA ${ }^{l}$, MARIA V. STEGANTSEVA ${ }^{l}$, OLGA V. ALEINIKOVA ${ }^{l}$ \\ ${ }^{1}$ Research Department, Belarusian Research Center for Pediatric Oncology, Hematology and Immunology, Minsk Region, Belarus \\ ${ }^{2}$ Prenatal Center, Belarusian Research and Practical Center "Mother and Child", Minsk, Belarus \\ ${ }^{3}$ BMT Department, Belarusian Research Center for Pediatric Oncology, Hematology and Immunology, Minsk Region, Belarus
}

\begin{abstract}
Nijmegen breakage syndrome (NBS) is a rare autosomal recessive disorder, characterized by spontaneous chromosomal instability with predisposition to immunodeficiency and cancer.

We present a repeated NBS in two sons from one woman after two marriages. We describe the clinical data, cytogenetic, and molecular findings of a prenatally diagnosed fetus, and his brothers with NBS. The first patient developed peripheral T-cell lymphoma at the age of 16 years and died 5 months after the protocol start. The diagnosis of NBS was established after his death. The second patient was born after the fifth pregnancy, third delivery in the second marriage; he developed cortical T-cell leukemia at the age of 3 years, received hematopoietic stem cells transplantation (HSCT) and he is alive now. In a year after repeated NBS case in this family, mother became pregnant again and the mutation was detected in the male fetus after the prenatal diagnosis; the pregnancy was aborted. At the age of 41 years, mother's seventh pregnancy finished by miscarriage. In three months, she was pregnant again, only one mutation in NBN gene was detected during the prenatal diagnostics in the female fetus; healthy female was born at term.

To our knowledge, this is the first time to describe the repeated cases of two patients born with Nijmegen breakage syndrome from one mother and two different fathers. This case highlights the value of checking NBN carrier in Belarusian families during genetic counselling.
\end{abstract}

Key words: Nijmegen breakage syndrome, repeated cases, lymphoma, leukemia, prenatal diagnosis, chromosomal instability.

(Centr Eur J Immunol 2020; 45 (4): 507-510)

\section{Introduction}

Nijmegen breakage syndrome (NBS) is an autosomal recessive chromosome instability disorder, characterized by growth and developmental defects (growth retardation, severe and progressive microcephaly, craniofacial features), immunodeficiency, high susceptibility to lymphoid malignancies, hypersensitivity to ionizing radiation and aberrant cell-cycle checkpoint control. The disease is caused by mutations in the $N B N$ gene, which encodes nibrin, a component of the complex involved in cellular response to DNA double-strand breaks [1-3].

Most of described patients are homozygous for a founder mutation in NBN gene (c.657del5), which leads to protein truncation. The disease is most common in people of Eastern European or Slavic background, specifically those from Poland, Southeast Germany, Czech Republic, and the Ukraine [3-6].
Herein, a repeated Nijmegen breakage syndrome is reported in two males from one mother after two marriages (Figure 1A) from the western part of Belarus and we describe the clinical data, cytogenetic, and molecular findings of a prenatally diagnosed fetus and his brothers with NBS. These data confirm the importance of genetic counseling of Slavic families and may substantiate the $N B N$ carrier detection in some regions of Eastern Europe.

\section{Patient 1 (P1)}

A 16-year-old male was admitted to the intensive care unit (ICU) with respiratory insufficiency and superior vena cava syndrome due to mediastinal lymphadenopathy at the level of the bifurcation of the trachea and thymomegalia. Peripheral T-cell lymphoma of grade III was established. Immunophenotyping of bone marrow aspirate showed

Correspondence: Svetlana O. Sharapova, Research Department, Immunology Laboratory, Belarusian Research Center for Pediatric Oncology, Hematology and Immunology, Minsk Region, Borovliani, Belarus, 223053, tel/fax: +375-17-265-42-22, e-mail: sharapovasv@ gmail.com Submitted: 03.01.2019; Accepted: 22.03.2019 
mature $\mathrm{T}$ cells phenotype $\left(\mathrm{CD}^{++} / \mathrm{CD}^{3+} / \mathrm{CD}^{++} / \mathrm{CD}^{++} /\right.$ $\left.\mathrm{CD}^{+}+/ \mathrm{TCRab}^{++}\right)$.

Initial chemotherapy for T-NHL was started, which consisted of CHOP-CHOP-ICE-DHAP-DHAP. During the lymphoma treatment, the diagnosis of NBS was not established and the patient received full dosage of anthracylines. The lymphoma progression was observed after the fifth course and the patient received DexaBEAM (BCNU, melphalan, vepesid, cytosar, and dexamethasone). On day 5 after block initiation, the patient was admitted to the ICU due to cytopenia, progressive respiratory insufficiency, systemic inflammatory response syndrome and oliguria. The patient died in two weeks due to multiple organ failure, polyserositis and lymphoma progression 5 months after the diagnosis.

\section{Patient 2 (P2), proband}

The third male was born at term after the fifth pregnancy in second marriage with microcephaly and typical craniofacial features. In spite of another mother's marriage, mutational analysis of $N B N$ gene revealed homozygous 5 base-pair deletion (del657A) after birth and resequencing of exon 5 of $N B N$ gene in the second husband, confirmed the heterozygous carrier (Fig. 1A).

P2 developed cortical T-ALL at the age of 3 years and received reduced doses of chemotherapy. During treatment, toxic hepatitis, steroid myopathy and amylase increasing were observed. He achieved a complete remission and was treated with hematopoietic stem cell transplant (HSCT) at the age of 4 years. After 350 postransplant days, severe complications were not observed.

\section{Prenatal diagnosis}

The first prenatal diagnosis was made at the 17 weeks of the third pregnancy after routine counseling due to the mother's age, with no signs of chromosomal instabilities being detected (Table 1, C1). A healthy boy was born. Later, sequencing of $N B N$ gene revealed a heterozygous deletion (Fig. 1A).

The most interesting results were obtained in the fetus F3 with confirmed NBS after sequencing. On the second day after taking chorionic villus sampling (CVS), the semi-direct method revealed normal male karyotype (trophoblast cells were investigated, 20 metaphases were analyzed). Long-term culture, when investigating cells of mesenchymal core of the villus, grew poorly, but 16 metaphases out of $40(40 \%)$ were found with a broad spectrum of abnormalities: supernumerary marker chromosomes, structural rearrangements (deletions and translocations) and chromosome breaks (Fig. 1B-1D, Table 1). Among them, the gaps were not found. Translocations and inversions of chromosomes 7 and 14 (with points of breakage in $7 \mathrm{p} 13,7 \mathrm{q} 35$ and $14 \mathrm{q} 11,14 \mathrm{q} 32$ ), characteristic of the culture of peripheral blood cells in NBS were absent. In two out of 40 metaphases, translocations involving chromosome 7 were identified, but the point of breakage was in region q22. Two metaphases with aberrant karyotype had the same translocation $\mathrm{t}(1 ; 12)(\mathrm{q} 42 ; \mathrm{q} 12)$ and additional abnormalities, which may possibly indicate the common origin and may be the result of a long cultivation. The sixth pregnancy was stopped by medical abortion.

Fetus 5 (F5) had normal karyotype and XX genotype; Sanger sequencing revealed only one mutation in $N B N$ gene (Fig. 1A).

Immunologic investigation for P1 was available only as detection of TRECs and KRECs on DNA isolated from the bone marrow aspirate at the time of lymphoma diagnosis before the chemotherapy start. The absence of TRECs ( 0 copies $/ 10^{6}$ mononuclear cells, normal range [NR] $>4000$ ) indicated severe abnormalities in T cellular immunity, deficiency in the number of precursors of B cells were detected too, by detection of KRECs $\left(0\right.$ copies $/ 10^{6}$ mononuclear cells, $\mathrm{NR}>1780$ ). Brother, $\mathrm{C} 2$, with only heterozygous c.657-661de15, had normal humoral and cellular parameters of the immune system. P2 had mild $\mathrm{T}$ cells lymphopenia $\left(\mathrm{CD}^{+}=1208\right.$ cells $\left./ \mu 1, \mathrm{NR} 1800-4600\right)$, since the age of one year he had decreased recent thymic emigrants $\left(\mathrm{CD} 4{ }^{+} \mathrm{CD} 45 \mathrm{RA}^{+} \mathrm{CD} 31^{+}=18.3 \%, \mathrm{NR} 40-70 \%\right)$ and hypogammaglobulinemia ( $\operatorname{IgG}=3.72 \mathrm{~g} / 1$ [NR 5-13 g/l], $\mathrm{IgA}=0.14 \mathrm{~g} / 1[\mathrm{NR} 0.2-1 \mathrm{~g} / 1]$ ). The number of TRECs $=$ 28670 was diminished, compared with healthy brother $\mathrm{C} 1$ $($ TRECs $=147878)$, but normal for healthy age range.

\section{Discussion}

Nijmegen breakage syndrome is a severe syndrome with a very high predisposition to malignancy. In an European study, presented predominantly Polish patients $(n=149)$ [6], and study of Russian cohort $(n=35)$ [7], cancer was noted in $42 \%$ and $57 \%$ of the patients before the age of 26 and 19 years, respectively, while $44 \%$ and $25 \%$ of all patients with malignancy had died due to progressive malignancy and/or complications of treatment $[6,7]$.

Nijmegen breakage syndrome should be suspected in pediatric patients with microcephaly presenting with a history of recurrent infections or lymphoma [8]. The first child of the described woman was not diagnosed during his lifetime and received a full course of chemotherapy for the treatment of lymphoma, which could be the cause of a fatal outcome. The literature describes cases of successful treatment of lymphoma in patients with NBS, using a reduced protocol [8], but Russian's cohort studies showed that $65 \%$ of malignant patients received conventional unmodified regimens and were well tolerated [7]. Cells from individuals with NBS have a decrease in colony-forming ability following exposure to ionizing radiation and radiomimetics in vitro, so the patients should be protected on radiological exposure. 

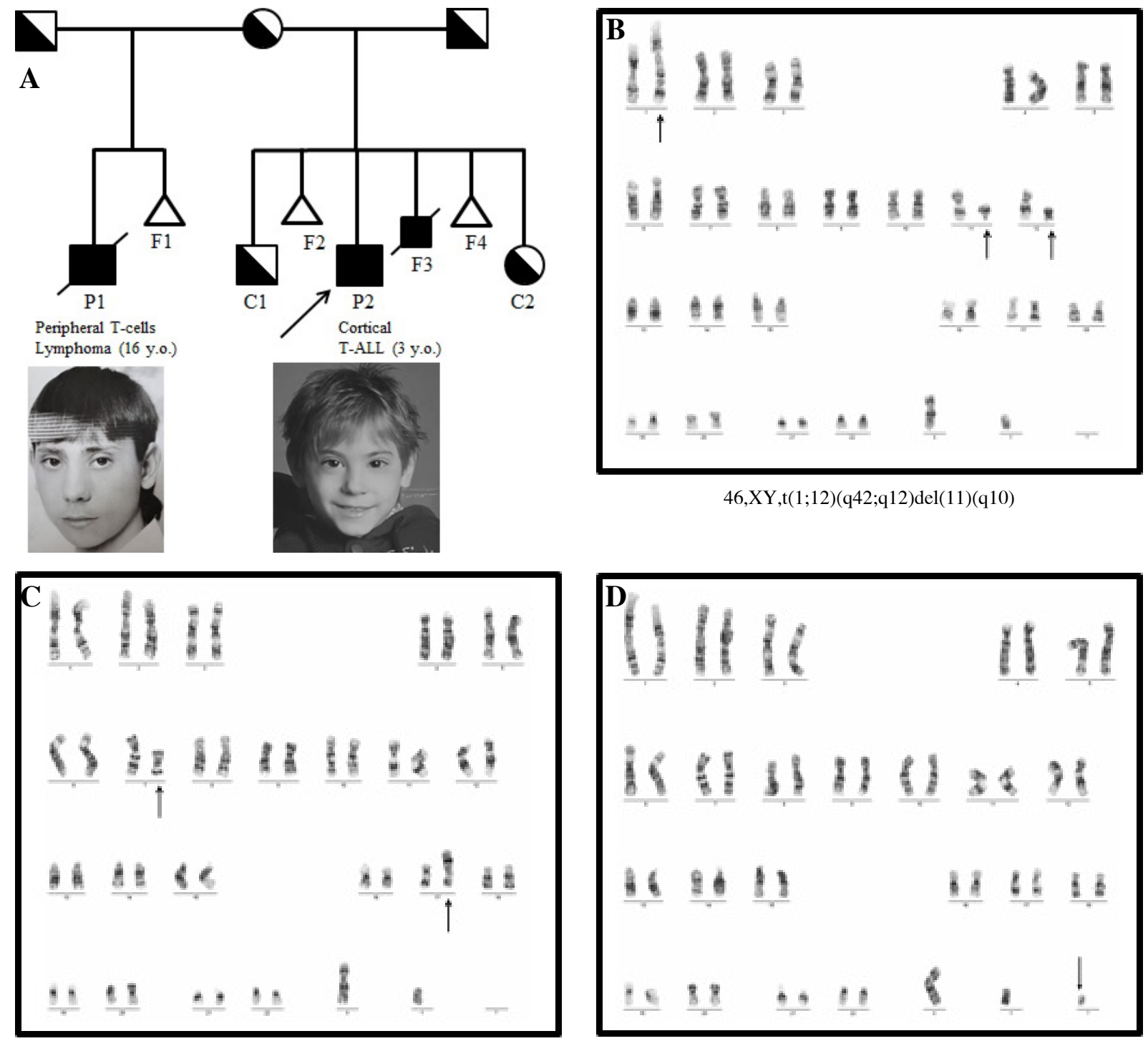

$46, X Y, t(7 ; 17)(q 22 ; p 13)$

$47, \mathrm{XY},+\mathrm{mar}$

Fig. 1. Pedigree of the family and G-banded karyogram of 3 metaphases from fetus F3. A) The symbols used are proband (arrow), male (box), female (circle), deceased (line through), affected male (black fill), unknown (no fill). P1 - the first son with NBS died of the lymphoma; F1 - fetus, nondeveloping pregnancy at 6 weeks, first marriage; C1 - carrier, healthy second son, second marriage; F2 - fetus, nondeveloping pregnancy at 6 weeks, second marriage; P2 - proband, the third son with NBS; F3 - male fetus with NBS, medical abortion; F4 - nondeveloping pregnancy; C5 - female fetus-carrier. B) G-banded karyogram of LTC of CVS showing translocation $(1 ; 12)$ and deletion (11)(q10). C) Two metaphases with 46,XY,t(7;17)(q22;p13) were detected out of 40 analyzed metaphases. D) Additional chromosome marker of unknown genesis was observed in 5 out of 40 analyzed metaphases

The cytogenetic data of different fetuses in one woman did not show important differences for the proportion of structural chromosomal abnormalities and breaks. Our results showed that specific chromosomal rearrangements that frequently involve chromosomes 7 and 14 of NBS patients were not found in metaphases of fetus F3. Spectrum of chromosomal abnormalities depends on the type of analyzed cells. Rearrangements of chromosomes 7 and
14 were usually detected in T-lymphocytes of NBS patients, which are represented in a sufficient amount in blood and bone marrow. Chromosomal aberrations in other cell lines, for example, in fibroblasts were different from those observed in T-cells. In some reports, balanced and unbalanced translocations, isochromosomes, partial endoreduplications, aneuploidies, and supernumerary marker chromosomes were described $[9,10]$. Genetic counseling 
Table 1. Karyotypes in AFC and CVC from Nijmegen breakage syndrome fetus

\begin{tabular}{ll}
\hline Fetus (material) & \multicolumn{1}{c}{ Karyotypes [number of metaphases] } \\
\hline C1 (AFC) & GTG-banding: 46, XY [20] \\
\hline P2 (AFC) & GTG-banding: 46, XY [10] \\
\hline F3 (CVC) & STC non-banded: 46, XY [20/20] \\
& LTC, GTG-banding: \\
& $46, \mathrm{XY}[24 / 40]$ \\
& $47, \mathrm{XY},+\operatorname{mar}[5 / 40]$ \\
& $46, \mathrm{XY}, \mathrm{t}(7 ; 17)(\mathrm{q} 22 ; \mathrm{p} 13)[2 / 40]$ \\
& $46, \mathrm{XY}, \mathrm{t}(1 ; 12)(\mathrm{q} 42 ; \mathrm{q} 12) \mathrm{del}(11)(\mathrm{q} 10)$ \\
& {$[1 / 40] 47, \mathrm{XY}, \mathrm{t}(1 ; 12)(\mathrm{q} 42 ; \mathrm{q} 12),+\mathrm{ace}$} \\
& {$[1 / 40] 48, \mathrm{XY}, \mathrm{del}(11)(\mathrm{p} 10),+2 \mathrm{mar}[1 / 40]$} \\
& $48, \mathrm{XY},+3 \operatorname{mar}[1 / 40]$ \\
& $46, \mathrm{XY}, \operatorname{chrb}(1)(\mathrm{p} 10)[1 / 40]$ \\
& $46, \mathrm{XY}, \operatorname{chtb}(18)(\mathrm{q} 21)[1 / 40]$ \\
& $46, \mathrm{XY}, \operatorname{chtb}(9)(\mathrm{q} 22)[1 / 40]$ \\
& $46, \mathrm{XY}, \operatorname{chtb}(8)(\mathrm{q} 13)[1 / 40]$ \\
& $46, \mathrm{XY}, \operatorname{chtb}(12)(\mathrm{q} 24.1)[1 / 40]$ \\
\hline
\end{tabular}

is an important aspect in the overall approach to families with primary immunodeficiencies. Prenatal diagnosis may prevent the birth of affected patients, or allow planning early and adequate therapeutic intervention. However, ethical consideration should be applied. Nowadays, Nijmegen breakage syndrome could not be completely cured, but the question of early transplantation of hematopoietic stem cells before the development of malignancies of the hematopoietic system is discussed in the medical literature [6-8].

While the $N B N$ c.657del5 mutation is found in all Slavic populations, a rather high heterozygote frequency $(0.5 \pm 1.0 \%)$ has been reported for Ukraine (Eastern Slavs), Poland, Czech Republic, Slovakia, and Sorbs in Germany (Western Slavs), and for Bulgaria (Southern Slavs) [4]. Carrier detection and genetic counseling programs have been very successful in reducing the birth prevalence of inherited disorders. These programs could be most successful when they are sensitive to the cultural backgrounds of populations in which they are applied. Despite Slavic people experienced massive population movement after World War II in Europe and population migration continued nowadays, our prospective study of NBS in Eastern Slavs indicated that West part of Ukraine and Belarus could be named as "East Slavic NBS geographical center" (Sharapova et al., submitted 2019); the presented family in this report was originated from West Belarus.

Investigation of Slavic population from Czech Republic proposed that heterozygous c.657del5 carriers have increased reproductive success [4], which could be supported by results of our study.

\section{Acknowledgments}

We thank all doctors for clinical help for patients and especially cytogeneticist Alena Valochnik. We are also grateful to Professor Nima Rezaei for critical reading of our manuscript.
We also appreciate the support of patient and his parents for agreeing to take part in study. The work was supported by the grants of Ministry of Health of Belarus.

\section{Ethics statement}

Informed consent was obtained from the members of the family who participated in this study. The authors are most grateful to the patients and their family members for their co-operation during this study and generous donation of samples and the patients' photo. The study was approved in accordance with the guidelines of the ethics committee of the Belarusian Research Center for Pediatric Oncology, Hematology and Immunology.

The authors declare no conflict of interest.

\section{References}

1. Weemaes CM, Hustinx TW, Scheres JM, et al. (1981): A new chromosomal instability disorder: the Nijmegen breakage syndrome. Acta Paed Scand 70: 557-564.

2. Digweed M, Sperling K (2004): Nijmegen breakage syndrome: clinical manifestation of defective response to DNA double-strand breaks. DNA Repair 3: 1207-1217.

3. Kondratenko I, Paschenko O, Polyakov A, Bologov A (2007): Nijmegen breakage syndrome. Adv Exp Med Biol 601: 61-67.

4. Seemanova E, Varon R, Vejvalka J, et al. (2016): The Slavic NBN founder mutation: a role for reproductive fitness? PLoS One 11: e0167984.

5. Varon R, Seemanova E, Chrzanowska K, et al. (2000): Clinical ascertainment of Nijmegen breakage syndrome (NBS) and prevalence of the major mutation, 657del5, in three Slav populations. Eur J Hum Gen 8: 900-902.

6. Wolska-Kusnierz B, Gregorek H, Chrzanowska K, et al. (2015): Nijmegen breakage syndrome: clinical and immunological features, long-term outcome and treatment options a retrospective analysis. J Clin Immunol 35: 538-549.

7. Deripapa E, Balashov D, Rodina Y, et al. (2017): Prospective study of a cohort of Russian Nijmegen breakage syndrome patients demonstrating predictive value of low kappadeleting recombination excision circle (KREC) numbers and beneficial effect of hematopoietic stem cell transplantation (HSCT). Front Immunol 8: 807.

8. Pastorczak A, Szczepanski T, Mlynarski W, International Berlin-Frankfurt-Munster (I-BFM) ALL host genetic variation working group (2016): Clinical course and therapeutic implications for lymphoid malignancies in Nijmegen breakage syndrome. Eur J Med Gen 59: 126-132.

9. Tupler R, Marseglia GL, Stefanini M, et al. (1997): A variant of the Nijmegen breakage syndrome with unusual cytogenetic features and intermediate cellular radiosensitivity. J Med Gen 34: 196-202.

10. Conley ME, Spinner NB, Emanuel BS, et al. (1986): A chromosomal breakage syndrome with profound immunodeficiency. Blood 67: 1251-1256. 